\title{
Catheter tips are a possible resource for biological study on catheter failure
}

\author{
Toshiaki Takahashi ${ }^{1}$, Takeo Minematsu ${ }^{2,3}$, Ryoko Murayama ${ }^{3,4}$, Gojiro Nakagami, \\ Taketoshi Mori ${ }^{1,3}$, Hiromi Sanada ${ }^{3,5, *}$ \\ ${ }^{1}$ Department of Life Support Technology (Molten), Graduate School of Medicine, The University of Tokyo, Tokyo, Japan; \\ ${ }^{2}$ Department of Skincare Science, Graduate School of Medicine, The University of Tokyo, Tokyo, Japan; \\ ${ }^{3}$ Division of Care Innovation, Global Nursing Research Center, Graduate School of Medicine, The University of Tokyo, Tokyo, \\ Japan; \\ ${ }^{4}$ Department of Advanced Nursing Technology, Graduate School of Medicine, The University of Tokyo, Tokyo, Japan; \\ ${ }^{5}$ Department of Gerontological Nursing/Wound Care Management, Graduate School of Medicine, The University of Tokyo, Tokyo, \\ Japan.
}

Summary Few studies have investigated the molecular mechanisms of catheter failure (CF). Herein, we performed histological and molecular biological analyses of the catheter tip to demonstrate its potential as a resource for biological investigation. Additionally, we searched for risk factors for the development of inflammation and coagulation, which are pathological conditions clarified by biological analysis. The CF group included 30 failed catheters involving thrombus and subcutaneous edema identified by ultrasonography. The No-CF group included 26 catheters with no complications. The removed catheter tips were fixed for hematoxylin-eosin (HE) staining with the application of a real-time reverse transcriptase polymerase chain reaction for eukaryotic $18 \mathrm{~S}$ ribosomal RNA (rRNA), interleukin $1 \beta$, tumor necrosis factor $\alpha$, tissue plasminogen activator, and plasminogen activator inhibitor 1 (SERPINE1). HE staining identified attached nuclear cells on the inner surfaces of both CF and No-CF catheters. The 18S rRNA was amplified in all samples. The expression level of SERPINE1 was significantly higher in the CF group than in the No-CF group $(p=0.01)$, whereas the expression levels of other genes did not differ between the groups. Symptoms of CF associated with the expression of SERPINE1 were analyzed. The catheter being in contact with blood vessels during placement was a suggested factor related to the high expression of SERPINE1 $(p=0.04)$. Catheter tips are a potential resource for biological investigation, and expression analysis of the attached cells can reflect the pathological condition of the catheterized tissue. Further studies using catheter tips are required to elucidate the molecular mechanisms of $\mathrm{CF}$.

Keywords: Catheter failure, catheter tips, histological analysis, thrombus

\section{Introduction}

Peripheral catheters are commonly used for the administration of fluids and medications. A recent study reported that more than $70 \%$ of all patients in

\footnotetext{
*Address correspondence to:

Dr. Hiromi Sanada, Department of Gerontological Nursing/ Wound Care Management, Graduate School of Medicine, University of Tokyo, Faculty of Medicine Bldg. No. 5-306, 7-3-1 Hongo, Bunkyo-ku, Tokyo 113-0033, Japan.

E-mail: hsanada-tky@umin.ac.jp
}

acute care hospitals use peripheral intravenous catheters (PIVCs) $(1,2)$. In addition, more than $30 \%$ of PIVCs are reportedly removed for unplanned reasons rather than replaced when clinically indicated, which is termed catheter failure (CF) $(3,4)$. Peripheral intravenous CF is a generic term used to refer to local complications associated with PIVCs, such as phlebitis and infiltration (5-7). CF is associated with clinical signs and symptoms, such as erythema, swelling, induration, bleeding and pain, and an insufficient infusion rate. CF negatively affects the patient's comfort and continuous fluid therapy. Catheter replacement, which 
is unavoidable in $\mathrm{CF}$, increases patient discomfort $(8,9)$, labor costs, and the cost of medical resources $(3,10$ 14). For these reasons, it is important to prevent $\mathrm{CF}$ in PIVCs and to pay special attention to signs, symptoms, and changes in the infusion rate.

The lack of effective preventive methods for CF may be due to insufficient investigation into the etiology of CF. Intravascular thrombus, subcutaneous edema, and catheter dislodgment have been previously discussed as possible etiologies of peripheral intravenous CF. Our previous study investigated the relationship between the etiology of CF confirmed by ultrasonography and the occurrence of peripheral intravenous CF (15). Notably, no catheter dislodgment was found among the cases of peripheral intravenous $\mathrm{CF}$. On the other hand, thrombus with subcutaneous edema was found to be significantly related to the occurrence of peripheral intravenous $\mathrm{CF}$.

In our previous study, we identified etiologies (dislodgment, thrombosis, and subcutaneous edema) by ultrasonography. Ultrasonography is useful to identify the structural changes at tissue or organ levels. However, it is very labor-intensive to perform an ultrasound examination just before removing a catheter at the bedside, especially difficult when doing at night or in situations where catheter removal is urgent. Here, instead, we wanted to focus on the removed catheter itself and to examine whether it is possible to clarify the condition of the blood vessels and surrounding tissue at the time of removal.

Therefore, we thought that we could explain the phenomenon of CF by the biological analysis of the removed catheter. With this new evaluation method, it would become possible to evaluate changes that are difficult to detect on the skin, and it may be possible to elucidate the causes of $\mathrm{CF}$ in detail and to evaluate the differences between varying devices and placement depths. Many studies have evaluated the status of catheter infection (16-19). For example, reports on the estimation of the infection route have been made by analyzing bacterial flora using next generation sequencing for the collected catheter (16). However, few studies have focused on the tissues and cells in the vicinity where the catheter is inserted.

Investigation at the cellular and molecular levels is essential for the development of an effective method to prevent CF. Here, we hypothesized that the cells, including erythrocytes, lymphocytes, vascular endothelial cells, and fibroblasts, attach to the inner and outer surfaces of the catheter, and they are a possible resource for molecular biological investigation.

The purpose of this preliminary study was to show the possibility of cytological and molecular biological investigation of the catheter tip by microscopic observation of the adhered host cells on the surface of the removed catheter tips and real-time reverse transcriptase polymerase chain reactions of housekeeping genes and genes related to inflammation and thrombosis. In this study, we searched for risk factors for the development of inflammation and coagulation, which are pathological conditions that can be clarified by biological analysis.

\section{Materials and Methods}

\subsection{Study setting and participants}

We recruited participants who had been admitted to a medical ward of a university hospital located in a metropolitan city in Japan who required PIVC for fluid therapy between January and June 2014. Any patients who received chemotherapy, were under 20 years of age, had a low cognitive level, or had an unstable physical condition were excluded. We repeatedly observed the patients who received PIVCs multiple times in order to include all PIVCs in this analysis. In this prospective observational study, we observed all PIVCs just before catheter removal. We defined PIVC with thrombus and subcutaneous edema detected on ultrasound and related to $\mathrm{CF}$ as "CF". We defined PIVC with no complications detected on ultrasound and no $\mathrm{CF}$ as "No-CF".

\subsection{Data collection procedure}

The patients' characteristics were collected either from medical records or from observations of the indwelling site before infusion therapy started. We asked the nurses to call us just before catheter removal. We then observed the signs and symptoms from macroscopic observation and observed the vessel lumen and surrounding tissues by ultrasound.

\subsection{Investigation items}

The researchers confirmed with the nurses the reasons for catheter removal. Accidental catheter removal was defined as CF. In this study, the ultrasound scanning technique was based on our previous study $(20,21)$. The motion images were recorded on a hard disk attached to the ultrasound equipment (Noblus ${ }^{\circledR}$; Hitachi Aloka Medical, Ltd., Tokyo, Japan), which included a lineararray (5.0-18.0 MHz) transducer. The identifications of thrombus, subcutaneous edema, and catheter tip position on ultrasound images were performed by a certified sonographer with over 10 years of experience. The sonographer was blinded to the condition of the PIVC.

The definitions of thrombus and subcutaneous edema were based on those of our previous study $(15,22)$. Intravenous thrombus was defined as a marked echogenic mass with an uneven surface. Final judgments were made using both transverse and longitudinal ultrasound images. Subcutaneous edema was defined by a homogeneous cobblestone appearance 
in the subcutaneous fat layer.

\subsection{PIVC collection}

PIVCs removed by the medical staff were collected. All catheters used in the target ward were Sure Shield Surflow II ${ }^{\circledR}$ (Terumo Corp., Tokyo, Japan). The catheter material was Teflon ${ }^{\circledR}$, and the length was $19-25 \mathrm{~mm}$. PIVC tips removed by a nurse (or researcher) were cut off at the base of the catheter with scissors, immersed in $1 \mathrm{ml} \mathrm{RNAlater}{ }^{\circledR}$ (Sigma-Aldrich Corp., MO, USA), and subsequently stored at $-20^{\circ} \mathrm{C}$.

\subsection{HE Staining}

The catheter tips were removed from the RNAlater and cut into two pieces longitudinally through the center of the tips. Both pieces of the catheter tip were placed in hematoxylin (Muto Pure Chemicals, Tokyo, Japan) for 2 minutes. Next, the pieces of the catheter were transferred to tap water and washed 5 times. Following incubation in hot water $\left(55^{\circ} \mathrm{C}\right)$ for 10 minutes, the catheter pieces were transferred to eosin (Muto Pure Chemicals Corp. Tokyo, Japan) for 10 seconds. Finally, the catheter pieces were dehydrated with a series of ethanol washes $(90 \%, 100 \%, 100 \%$, and $100 \%)$, and stored in $100 \%$ ethanol. The pieces of the catheter tips were placed in a droplet of glycerin and observed with an optical microscope.

\subsection{Real time reverse transcription polymerase chain reaction (real-time $R T$-PCR)}

Total RNA was extracted using a RNeasy Plus Mini Kit (QIAGEN, Venlo, Netherlands). The pieces of the catheter tips were immersed in a lysis buffer and flushed repeatedly with a $25 \mathrm{G}$ needle. Next, RNA was extracted according to the manufacturer's instructions. Reverse transcription was carried out by the $\mathrm{TM} 100^{\mathrm{TM}}$ Thermal Cycler (Bio-Rad, Hercules, CA) and the High Capacity cDNA Reverse Transcription Kit (Thermo Fisher Scientific, Waltham, MA). Real-time RT-PCR was performed with a real-time PCR system (Agilent, Santa Clara, CA) and TaqMan Gene Expression assays (Thermo Fisher Scientific) for tumor necrosis factor $\alpha$ $(T N F)$, interleukin-1 $\beta$ (IL1B), serpin family E member 1 ((SERPINE1) plasminogen activator inhibitor 1), and tissue plasminogen activator (PLAT). ILIB and TNF were biomarkers for inflammation, and PLAT and SERPINE1 were biomarkers for thrombosis. As an internal standard, 18S ribosomal RNA (rRNA) was simultaneously amplified. Each reaction was performed in triplicate.

At first, the amplifications were qualitatively evaluated. Samples showed typical amplification curves, and any cycle threshold $(\mathrm{Ct})$ values were considered as "detected". Samples with Ct values of 35 or lower were evaluated as "quantified". Subsequently, qualitative analysis was conducted only in the quantified samples. Relative expression levels of target genes (TNF, $I L 1 B, S E R P I N E 1$, and PLAT) were calculated by the comparative $\mathrm{Ct}$ method with the following formulas and the $\Delta \Delta \mathrm{Ct}$ values were used for the statistical analysis.

$$
\begin{aligned}
& \Delta C t=(C t \text { target gene }-C t 18 S r R N A) \\
& \Delta \Delta C t=\Delta C t-\text { Average of } \triangle C t C F \text { negative } \\
& \text { Relative expression level }=2^{(-\Delta \Delta C t)}
\end{aligned}
$$

\subsection{Statistical analyses}

Results are presented as mean \pm standard deviation. Statistical differences between the CF and No-CF groups were determined using Student's $t$-test or the rank sum test. For risk factor analysis, we compared $\mathrm{CF}$ with high gene expression $(\Delta \Delta C t>1)$ of biomarkers and No-CF with low gene expression $(\Delta \Delta C t<1)$ of biomarkers. A $p$ value of $<0.05$ was considered statistically significant. All statistical analyses were performed using STATA/SE 15.0 software (StataCorp LP, College Station, TX, USA).

\subsection{Ethical considerations}

The study protocol was approved by the Research Ethics Committee of The University of Tokyo (\#10348). Written informed consent was obtained from all patients or their proxies prior to participation in the study.

\section{Results}

\subsection{Participants and PIVC characteristics}

In this study, the researchers investigated 186 catheters using an ultrasonography device just before catheter removal. The study included $26 \mathrm{CF}$ patients whose PIVC had thrombus with subcutaneous edemarelated $\mathrm{CF}$ and 30 No-CF patients whose PIVC had no complications. Thirty of the 56 participants were men. The patients' mean age (SD) was $69.1( \pm 12.8)$ years, and the mean body mass index was $22.4( \pm 3.5) \mathrm{kg} / \mathrm{m}^{2}$ (Table 1).

\subsection{Presence of adhered cells}

HE staining showed the presence of adhered cells on the surface of the catheter tips. Erythrocytes, which have no nucleus, were stained only with eosin, and a double positive for hematoxylin and eosin revealed the presence of nucleated cells, such as white blood cells, endothelial cells, and fibroblasts. In all samples, a large number of erythrocytes and nucleated cells were observed only on the inner surface of the catheter tips. The morphology of the nucleated cells was round 
in shape, suggesting that they were white blood cells (Figure 1).

\subsection{Expression analysis}

Expression of $18 S r R N A$, an internal standard, was examined by real-time RT-PCR. All samples showed the specific amplification curves and their $\mathrm{Ct}$ values less than 35. The median (interquartile) $\mathrm{Ct}$ value of $18 \mathrm{~S}$ $r R N A$ were 22.4 (19.7-24.5) and 24.5 (20.6-27.9) in CF and NO-CF groups, respectively $(p=0.06)$.

The expression of target genes was qualitatively evaluated (Figure 2). The proportion of the detected samples for TNF, IL1B, SERPINE1, and PLAT was

Table 1. Participants and PIVC characteristics

\begin{tabular}{lcccc}
\hline Items & No-CF & $n=30$ & CF & $n=26$ \\
\hline Age; years* & 69.8 & $(12.0)$ & 68.3 & $(12.0)$ \\
Sex & 17 & $(56.7)$ & 13 & $(50.0)$ \\
BMI* & 22.6 & $(3.2)$ & 22.3 & $(3.7)$ \\
History of present illness & & & & \\
$\quad$ Neoplasms & 18 & $(60.0)$ & 14 & $(53.8)$ \\
$\quad$ Digestive system & 25 & $(83.3)$ & 20 & $(76.9)$ \\
$\quad$ Certain infectious & 4 & $(13.3)$ & 1 & $(3.8)$ \\
Diabetes & 9 & $(30.0)$ & 7 & $(26.9)$ \\
Anticoagulant drug use & 4 & $(13.3)$ & 1 & $(3.8)$ \\
Catheter size & & & & \\
$\quad$ 22G & 22 & $(73.3)$ & 22 & $(84.6)$ \\
$\quad 24 \mathrm{G}$ & 8 & $(26.7)$ & 4 & $(15.4)$ \\
Catheter anatomical site (forearm) & 28 & $(93.3)$ & 25 & $(96.2)$ \\
Catheter dwell time* & 65.0 & $(32.0)$ & 53.6 & $(27.4)$ \\
\hline
\end{tabular}

$\mathrm{N}(\%)$, * mean (SD). BMI: body mass index; CF: catheter failure; PIVC: peripheral intravenous catheters.
$75 \%$ (42/56), 94.6\% (53/56), 98.2\% (55/56), and 19.6\% (11/56), respectively. The proportion of the quantified samples for TNF, IL1B, SERPINE1, and PLAT was $64.3 \%(36 / 56), 60.7 \%(34 / 56), 71.4 \%$ (40/56), and $7.1 \%$ (4/56), respectively.

\subsection{Effects of the number of adhered cells on expression analysis}

In order to confirm the effect of the number of adhered cells on the quantification of target gene expression, the number of adhered cells and the expression level of $18 S$ rRNA were compared between quantified and not quantified samples for $T N F$. The median (interquartile) numbers of adhered cells were 21.3 (19.7-23.2) and $27.4(25.3-29.0)$ in the quantified and the not quantified groups, respectively $(p=0.01)$.

\subsection{Comparison between the CF and No-CF groups}

Tables 2 and 3 show the proportion of detected and quantified samples for the target genes, respectively. There was no significant difference in the proportion of detected samples for all genes between groups. The frequency of quantified samples was significantly high in ILIB and SERPINE1 and tended to be high in TNF in the CF group compared with those in the No-CF group. In other words, it suggests that whether a sample is quantified or not depends on the number of cells that can be collected.

The expression levels of target genes only in the quantified samples were compared between the CF and
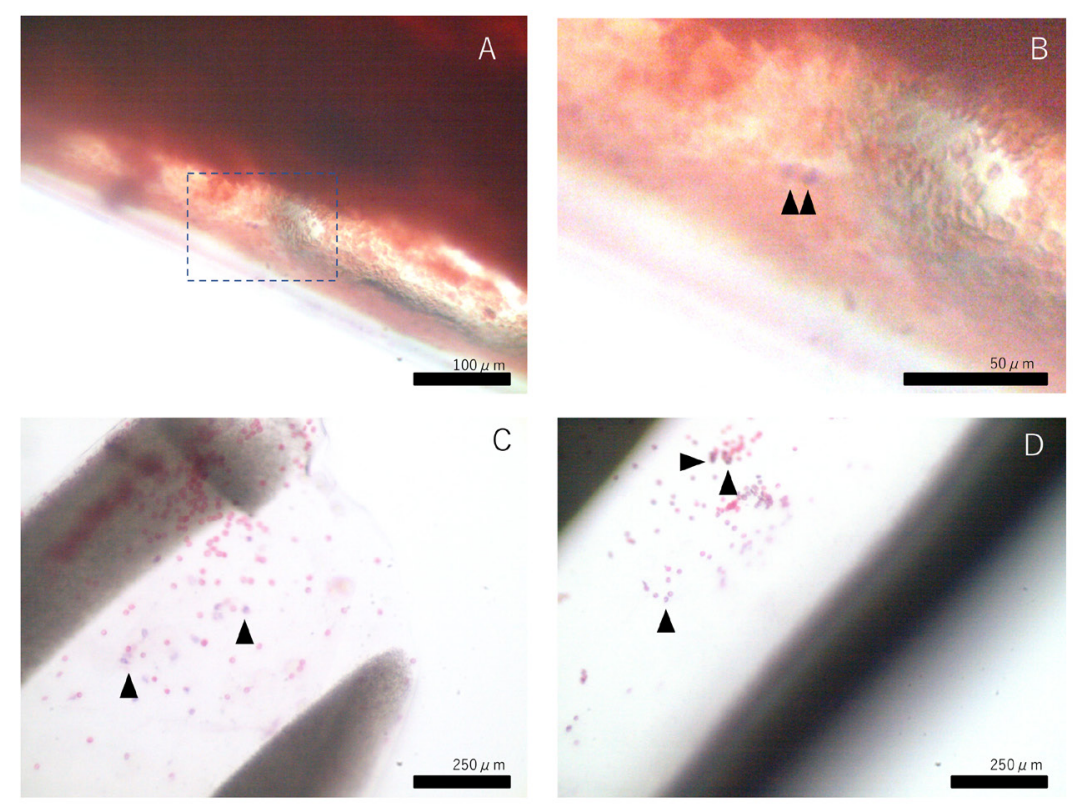

Figure 1. Hematoxylin-eosin staining of catheter tips. A few, round, nucleated cells (arrowheads) were observed among many enucleated cells. Two catheters are represented: $\mathbf{A}$ and $\mathbf{B}$ are images of one catheter, and $\mathbf{C}$ and $\mathbf{D}$ are images of another catheter. In $\mathbf{A}$ and $\mathbf{B}$, many red blood cell aggregates and some cells with nuclei are present; $\mathbf{B}$ is an enlarged image of the broken line portion of $\mathbf{A}$. Panels $\mathbf{C}$ and $\mathbf{D}$ show some cells adhering to the lumen of the catheter. 


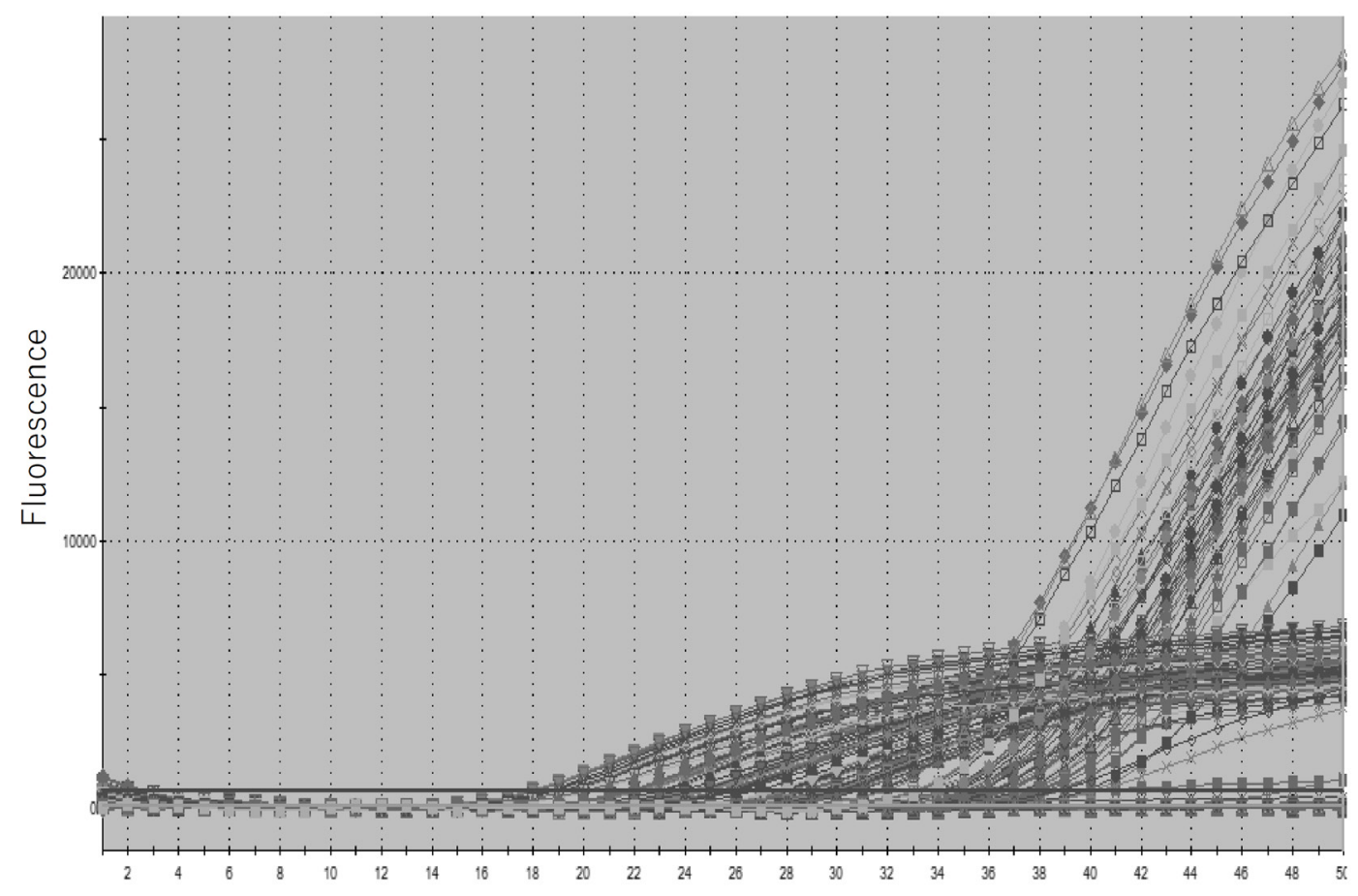

Figure 2. Amplification curve.

Table 2. The proportion of the detected samples for target genes

\begin{tabular}{lccl}
\hline Detected & No-CF $30 N(\%)$ & CF $26 N(\%)$ & $p$ value \\
\hline TNF & $20(66.7)$ & $22(84.6)$ & 0.112 \\
IL1B & $27(90.0)$ & $26(100.0)$ & 0.097 \\
SERPINE1 & $29(96.7)$ & $26(100.0)$ & 0.348 \\
PLAT & $5(16.7)$ & $6(23.1)$ & 0.547 \\
& & & $\chi^{2}$ test \\
\hline
\end{tabular}

CF: Catheter failure; TNF: tumor necrosis factor; IL1B: interleukin-1B; SERPINE1: serpin family E member 1; PLAT: plasminogen activator, tissue type.

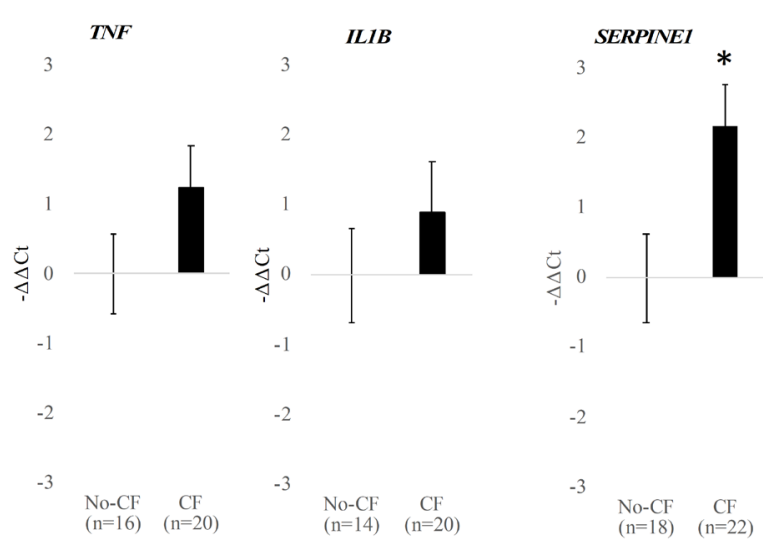

Figure 3. Comparison of expression levels of target genes between the catheter failure (CF) and No-CF groups. Data are presented as means \pm Standard Division of $-\Delta \Delta$ Ct. ${ }^{*} p<0.05$.

No-CF groups (Figure 3 ). The $-\Delta \Delta \mathrm{Ct}$ values of $T N F$ expression were $1.24 \pm 0.60$ in the $\mathrm{CF}$ group and $0.0 \pm$
Table 3. The proportion of the quantified samples for target genes

\begin{tabular}{lcrl}
\hline Quantified & No-CF 30 N(\%) & CF 26 N(\%) & $p$ value \\
\hline TNF & $16(53.3)$ & $20(76.9)$ & 0.066 \\
IL1B & $14(46.7)$ & $20(76.9)$ & 0.021 \\
SERPINE1 & $18(60.0)$ & $22(84.6)$ & 0.042 \\
PLAT & $2(06.7)$ & $2(07.7)$ & 0.882 \\
& & & $\chi^{2}$ test \\
\hline
\end{tabular}

CF: Catheter failure; TNF: tumor necrosis factor; IL1B: interleukin-1 $\beta$; SERPINE1: serpin family E member 1; PLAT: plasminogen activator, tissue type.

0.57 in the No-CF group $(p=0.15)$. The $-\Delta \Delta C t$ values of $I L 1 B$ expression were $0.90 \pm 0.73$ in the CF group and $0.0 \pm 0.68$ in the No-CF group $(p=0.39)$. The $-\Delta \Delta \mathrm{Ct}$ values of SERPINE1 expression were $2.18 \pm 0.60$ in the $\mathrm{CF}$ group and $0.0 \pm 0.63$ in the No-CF group $(p<0.01)$. The $-\triangle \Delta \mathrm{Ct}$ values of PLAT expression were $-8.15 \pm$ 6.04 in the CF group and $0.0 \pm 0.66$ in the No-CF group. Statistical analysis was not performed because only two samples were confirmed the expression of PLAT.

The risk factors for CF that have been identified in previous studies were compared between SERPINE1 expression groups (Table 4). As a result, the catheter coming into contact with blood vessels during placement was suggested as a factor related to the high expression of SERPINE1 $(p=0.04)$ rather than any other patient characteristics.

\section{Discussion}

To the best of our knowledge, this is the first study to 
Table 4. The proportion of the quantified samples for target genes

\begin{tabular}{|c|c|c|c|c|c|}
\hline Items & $\begin{array}{l}\text { No-CF with } S E R P I N E 1<1 \\
\text { mean } / \mathrm{n}\end{array}$ & $\begin{array}{c}n=12 \\
(\mathrm{SD}) /(\%)\end{array}$ & $\begin{array}{c}\text { CF with } \operatorname{SERPINE} 1>1 \\
\text { mean } / \mathrm{n}\end{array}$ & $\begin{array}{c}n=14 \\
(\mathrm{SD}) /(\%)\end{array}$ & $p$ \\
\hline Age (y) & 63.5 & $(12.8)$ & 65.6 & $(15.3)$ & 0.71 \\
\hline Sex (male) & 8 & $(66.7)$ & 9 & $(64.3)$ & 0.90 \\
\hline BMI & 21.6 & $(3.1)$ & 21.8 & $(3.1)$ & 0.89 \\
\hline Disease (digestive system disease) & 11 & $(91.7)$ & 12 & $(85.7)$ & 0.89 \\
\hline Disease (neoplastic) & 9 & $(75.0)$ & 8 & $(57.1)$ & 0.91 \\
\hline Oral administration of anticoagulant & 3 & $(25.0)$ & 1 & $(7.1)$ & 0.21 \\
\hline Part (forearm) & 12 & $(100.0)$ & 13 & $(92.9)$ & 0.89 \\
\hline Gauge (22G) & 9 & $(75.0)$ & 13 & $(92.9)$ & 0.21 \\
\hline Heparin lock time more than 12 hours & 9 & $(75.0)$ & 12 & $(85.7)$ & 0.24 \\
\hline Irritant drug & 1 & $(8.3)$ & 3 & $(21.4)$ & 0.36 \\
\hline Antibacterial drug & 5 & $(41.7)$ & 9 & $(64.3)$ & 0.25 \\
\hline Total dose of drug solution (mL) & $2,165.0$ & $(2,179.8)$ & $2,464.9$ & $(3,121.6)$ & 0.78 \\
\hline Heparin lock total time (h) & 36.5 & $(26.0)$ & 20.8 & $(17.7)$ & 0.09 \\
\hline Contact with the blood vessels by catheter & 0 & $(0.0)$ & 4 & $(28.6)$ & $0.04 *$ \\
\hline Blood vessel diameter & 2.45 & $(1.0)$ & 2.55 & $(0.8)$ & 0.79 \\
\hline
\end{tabular}

CF: catheter failure; SERPINE1: serpin family E member 1; BMI: body mass index.

biologically analyze the extracted PIVC. Our results suggest that catheter tips are a potential resource for biological investigation, and expression analysis of the attached cells can reflect the pathological condition of the catheterized tissue. Further studies using catheter tips are required to elucidate the molecular mechanisms of CF. Furthermore, we performed an analysis of SERPINE1, which was related to CF. These results suggested that in cases with high expression of SERPINE1, the catheter may stimulate the blood vessel wall by coming in contact with it. Keeping this in mind could be useful when considering future CF precautions.

There were three main findings from this study. The first finding was the presence of the attached humanderived cells on the inside surface of the removed catheter tips. Prior research has reported that blood in the lumen of the catheter is coagulated at the catheter tip if there is no infusion (23). In particular, it has been shown that backflow of blood occurs owing to the locking procedure performed when the catheter use is interrupted (24-26). In the wards covered in this study, a needleless connector was used to connect the infusion tube, and a positive pressure heparin flush was performed at least once every 24 hours. Nevertheless, HE staining and the amplification of $18 S r R N A$ indicated the adherence of abundant enucleated erythrocytes and nucleated cells on the inner surface of tips even in the No-CF catheters.

Secondly, analysis of the cells attached to the removed catheter showed that SERPINE 1 mRNA, which is an inhibitor of thrombus removal, was highly expressed in the CF group as compared to the NoCF group. Although this mRNA may be derived from vascular endothelial cells, we believe that the most likely candidate may be derived from monocytes (27). SERPINE1 is expressed by various kinds of cells, including endothelial cells, monocytes, hepatocytes, granulosa cells, and vascular smooth muscle cells, and inhibits the activities of urinary-type and tissue-type plasminogen activators (28). Performing catheterization is foreign to the human body, and the coagulation reaction is caused by disturbance of all three factors of Virchow's triad (29). Especially in the distal end of the catheter, a fibrin sheath is formed. Erythrocytes and monocytes are entangled by the fibrin sheath, and thrombus is formed. In this study, the monocytes may express SERPINE1 and may inhibit thrombus removal and promote thrombus formation. A significant difference was not detected between $I L 1 B$ and $T N F$, as hypothesized by the present study. This may be owing to the fact that vascular endothelial cells and surrounding tissues could not be collected. Vascular endothelial cells and surrounding tissues should have been in contact with the catheter, but it is thought that these were scraped off in the catheter removal process. $P A L T$ is a marker closely related to SERPINE1 $(30,31)$, but its expression was not observed in most samples in this study. This may be owing to PALT being tissue derived rather than monocyte derived. In the future, it may be better to verify the chemotactic factors of MF and monocytes as inflammation markers.

Finally, CF associated with the expression of SERPINE1 was analyzed. The catheter being in contact with blood vessels during placement was suggested to be a factor related to high expression of SERPINE1 ( $p=$ $0.04)$. Mechanical stimulation of the blood vessel wall by a catheter is a risk factor for $\mathrm{CF}$ that has been pointed out in our previous studies (32). It is suggested that inflammation by simulation occurs when the tip of the catheter contacts the vascular endothelial cells (33). This study suggested that neutrophils were more aggregated in the fibrin sheath, causing coagulation to progress further.

Some studies have been conducted in the past to detect infection using removed catheters. We believed that our method by real time RT-PCR would contribute to the detection of coagulation. As mentioned in the 
background section, we performed ultrasonography just before catheter removal to detect the etiology of catheter failure. This allowed real-time assessment and direct observation of the blood vessel; however, it may be difficult to conduct ultrasonography at night or in situations where removal is urgent. In such situations, conventional analysis is useful to detect the etiology of CF. Our suggested technique might be useful to evaluate new vascular access devices, fixation devices, or other medical devices.

This study showed that it is possible to elucidate a biological cause of CF. Furthermore, real-time RT-PCR, using cells extracted from catheters after removal, may be a useful new method to evaluate peripheral venous catheters. Our findings suggest that catheter contact is related not only to inflammation but also to coagulation. This result will be useful for devising and evaluating future measures to prevent $\mathrm{CF}$ in clinical nursing. The prevention of mechanical stimuli for the prevention of $\mathrm{CF}$ has been advocated in recent years (32-34). Since this study suggested that the catheter tip has an effect on the blood vessel wall and coagulation, it may be advisable that clinical nurses need to pay attention to the fixation of the catheter for confirmation. Confirmation of catheter position by ultrasonography is likely to be effective for the prevention of $\mathrm{CF}$, and future clinical application is expected.

There were limitations to the variations of clinical samples dealt with in this study, and only one type of catheter was used. In order to extrapolate these results, it is necessary to consider the type and coating of the catheter. Furthermore, peripheral intravenous catheter infections have been reported (35) and may have been associated with CF. However, we did not include cases in which the target catheter was diagnosed as an infection source. Factor analysis of SERPINE1 expression in this study could not be performed using multivariate analysis owing to the small sample size. However, a relationship between SERPINE1 and vessel wall contact can be suggested because this was not related to patient factors such as oral anticoagulant administration or age.

Our findings suggest that catheter tips are a potential resource for biological investigation, and expression analysis of the attached cells can reflect the pathological condition of the catheterized tissue. This result implicates the involvement of SERPINE1 in drip failure and pain as a new pathological condition. In addition, the etiology of SERPINE1 expression was suggested to be mechanical stimulation by the catheter coming into contact with the vessel wall. Further studies using catheter tips are required to elucidate the molecular mechanisms of CF.

\section{Acknowledgements}

This work was conducted as a part of a project funded by the JSPS KAKENHI Grant Number 26670915 and 18074373.

\section{References}

1. Zingg W, Pittet D. Peripheral venous catheters: an underevaluated problem. Int J Antimicrob Agents. 2009; 34 (Suppl 4):S38-42.

2. Waitt C, Waitt P, Pirmohamed M. Intravenous therapy. Postgrad Med J. 2003; 80:1-6.

3. Rickard CM, Webster J, Wallis MC, Marsh N, McGrail MR, French V, Foster L, Gallagher P, Gowardman JR, Zhang L, McClymont A, Whitby M. Routine versus clinically indicated replacement of peripheral intravenous catheters: a randomised controlled equivalence trial. Lancet. 2012; 380:1066-1074.

4. Murayama R, Uchida M, Oe M, Takahashi T, Oya M, Komiyama C, Sanada H. Patient risk factors for developing sign- and symptom-related peripheral intravenous catheter failure: A retrospective study. J Jpn WOCM. 2015; 19:13594-13402.

5. Gorski LA. Infusion nursing standards of practice. J Infus Nurs. 2007; 30:20-21.

6. Pujol M, Hornero A, Saballs M, Argerich MJ, Verdaguer R, Cisnal M, Pena C, Ariza J, Gudiol F. Clinical epidemiology and outcomes of peripheral venous catheter-related bloodstream infections at a universityaffiliated hospital. J Hosp Infect. 2007; 67:22-29.

7. Dougherty L. IV therapy: recognizing the differences between infiltration and extravasation. Br J Nurs. 2008; 17:896-891.

8. Bregenzer T, Conen D, Sakmann P, Widmer AF. Is routine replacement of peripheral intravenous catheters necessary? Arch Intern Med. 1998; 158:151-156.

9. Xu L, Hu Y, Huang X, Fu J, Zhang J. Clinically indicated replacement versus routine replacement of peripheral venous catheters in adults: A nonblinded, clusterrandomized trial in China. Int J Nurs Pract. 2017; 23(6). doi: 10.1111/ijn.12595.

10. Ansel B, Boyce M, Embree JL. Extending short peripheral catheter dwell time: A best practice discussion. J Infus Nurs. 2017; 40:143-146.

11. Rickard CM, Marsh NM, Webster J, et al. Intravascular device administration sets: replacement after standard versus prolonged use in hospitalised patients-a study protocol for a randomised controlled trial (The RSVP Trial). BMJ Open. 2015; 5:e007257.

12. Rickard CM, McCann D, Munnings J, McGrail MR. Routine resite of peripheral intravenous devices every 3 days did not reduce complications compared with clinically indicated resite: a randomised controlled trial. BMC Med. 2010; 8:53.

13. Webster J, Osborne S, Rickard CM, New K. Clinicallyindicated replacement versus routine replacement of peripheral venous catheters. Cochrane Database Syst Rev. 2013; 1:CD007798.

14. Tuffaha HW, Rickard CM, Webster J, Marsh N, Gordon L, Wallis M, Scuffham PA. Cost-effectiveness analysis of clinically indicated versus routine replacement of peripheral intravenous catheters. Appl Health Econ Health Policy. 2014; 12:51-58.

15. Takahashi T, Murayama R, Oe M, Nakagami G, Tanabe H, Yabunaka K, Arai R, Komiyama C, Uchida M, Sanada H. Is thrombus with subcutaneous edema detected by 
ultrasonography related to short peripheral catheter failure? A prospective observational study. J Infus Nurs. 2017; 40:313-322.

16. Choudhury MA, Marsh N, Banu S, Paterson DL, Rickard CM, McMillan DJ. Molecular comparison of bacterial communities on peripheral intravenous catheters and matched skin swabs. PLoS One. 2016; 11:e0146354.

17. Choudhury MA, Sidjabat HE, Rathnayake IU, Gavin N, Chan RJ, Marsh N, Banu S, Huygens F, Paterson DL, Rickard CM, McMillan DJ. Culture-independent detection of chlorhexidine resistance genes qacA/B and smr in bacterial DNA recovered from body sites treated with chlorhexidine-containing dressings. J Med Microbiol. 2017; 66:447-453.

18. Cicolini G, Manzoli L, Simonetti V, Flacco ME, Comparcini D, Capasso L, Di Baldassarre A, Eltaji Elfarouki G. Phlebitis risk varies by peripheral venous catheter site and increases after 96 hours: a large multicentre prospective study. J Adv Nurs. 2014; 70:25392549.

19. Cinel I, Dellinger RP. Guidelines for severe infections: are they useful? Curr Opin Crit Care. 2006; 12:483-488.

20. Tanabe H, Murayama R, Yabunaka K, Oe M, Takahashi T, Komiyama C, Sanada H. Low-angled peripheral intravenous catheter tip placement decreases phlebitis. J Vasc Access. 2016; 17:542-547.

21. Tanabe H, Takahashi T, Murayama R, Yabunaka K, Oe M, Matsui Y, Arai R, Uchida M, Komiyama C, Sanada H. Using Ultrasonography for vessel diameter assessment to prevent infiltration. J Infus Nurs. 2016; 39:105-111.

22. Yabunaka K, Takahashi T, Tanabe H, Kawamoto A, Oe M, Arai R, Sanada H. Ultrasonographic appearance of infusion via the peripheral intravenous catheters. J Nurs Sci Eng. 2015; 2:40-46.

23. Baekgaard N, Broholm R, Just S, Jorgensen M, Jensen LP. Long-term results using catheter-directed thrombolysis in 103 lower limbs with acute iliofemoral venous thrombosis. Eur J Vasc Endovasc Surg. 2010; 39:112-117.

24. Keogh S, Flynn J, Marsh N, Mihala G, Davies K, Rickard C. Varied flushing frequency and volume to prevent peripheral intravenous catheter failure: a pilot, factorial randomised controlled trial in adult medicalsurgical hospital patients. Trials. 2016; 17:348.

25. Hamze B, Vaillancourt R, Sharp D, Villarreal G. A comparison of taste and odor perception in pediatric patients receiving a $0.9 \%$ sodium chloride flush from 2 different brands of prefilled $0.9 \%$ sodium chloride syringes. J Infus Nurs. 2016; 39:18-24.

26. Upadhyay A, Verma KK, Lal P, Chawla D, Sreenivas V. Heparin for prolonging peripheral intravenous catheter use in neonates: a randomized controlled trial. J Perinatol. 2015; 35:274-277.

27. Hamilton JA, Whitty GA, Wojta J, Gallichio M, McGrath $\mathrm{K}$, Ianches $\mathrm{G}$. Regulation of plasminogen activator inhibitor-1 levels in human monocytes. Cell Immunol. 1993; 152:7-17.

28. Sprengers ED, Kluft C. Plasminogen activator inhibitors. Blood. 1987; 69:381-387.

29. Kyrle PA, Eichinger S. Is Virchow's triad complete? Blood. 2009; 114:1138-1139.

30. Cao C, Lawrence DA, Li Y, Von Arnim CA, Herz J, Su EJ, Makarova A, Hyman BT, Strickland DK, Zhang L. Endocytic receptor LRP together with tPA and PAI-1 coordinates Mac-1-dependent macrophage migration. EMBO J. 2006; 25:1860-1870.

31. Nassar T, Akkawi S, Shina A, Haj-Yehia A, Bdeir K, Tarshis M, Heyman SN, Higazi AA. In vitro and in vivo effects of tPA and PAI-1 on blood vessel tone. Blood. 2004; 103:897-902.

32. Murayama R, Takahashi T, Tanabe H, Yabunaka K, Oe M, Oya M, Uchida M, Komiyama C, Sanada H. The relationship between the tip position of an indwelling venous catheter and the subcutaneous edema. Biosci Trends. 2015; 9:414-419.

33. Piper R, Carr PJ, Kelsey LJ, Bulmer AC, Keogh S, Doyle BJ. The mechanistic causes of peripheral intravenous catheter failure based on a parametric computational study. Sci Rep. 2018; 8:3441.

34. Rickard CM, Marsh N, Webster J, et al. Dressings and securements for the prevention of peripheral intravenous catheter failure in adults (SAVE): A pragmatic, randomised controlled, superiority trial. Lancet. 2018; 392:419-430.

35. Ray-Barruel G. Infection prevention: Peripheral intravenous catheter assessment and care. Aust Nurs Midwifery J. 2017; 24:34.

(Received September 30, 2019; Revised October 24, 2019; Accepted October 26, 2019) 